\title{
The Misfit Dislocation Core Phase in Complex Oxide Heteroepitaxy
}

Núria Bagués, José Santiso, Bryan D. Esser, Robert E.A. Williams, Dave W. McComb, Zorica Konstantinovic, Lluís Balcells, and Felip Sandiumenge*

Dr. N. Bagués, Dr. Ll. Balcells, Dr. F.Sandiumenge

Institut de Ciència de Materials de Barcelona, ICMAB-CSIC

Campus de la UAB, 08193 Bellaterra, Catalonia, Spain.

E-mail: felip@icmab.cat

Dr. N. Bagués, Dr. J. Santiso

Catalan Institute of Nanoscience and Nanotechnology (ICN2), CSIC and BIST

Campus UAB, Bellaterra, 08193 Barcelona, Catalonia, Spain.

Dr. B.D. Esser, Dr. R.E.A. Williams, Prof. D.W. McComb

Center for Electron Microscopy and Analysis

The Ohio State University, Columbus, USA.

Dr. Z. Konstantinovic

Center for Solid State Physics and New Materials, Institute of Physics Belgrade, University of Belgrade, Pregrevica 118, 11080 Belgrade, Serbia.

Keywords: complex oxides, epitaxy, misfit dislocations, oxygen vacancies, strain

Misfit dislocations form self-organized nanoscale linear defects exhibiting their own distinct structural, chemical, and physical properties which, particularly in complex oxides, hold a strong potential for the development of nanodevices. However, the transformation of such defects from passive into potentially active functional elements necessitates a deep understanding of the particular mechanisms governing their formation. Here we combine different atomic resolution imaging and spectroscopic techniques to determine the complex structure of misfit dislocations in the perovskite type $\mathrm{La}_{0.67} \mathrm{Sr}_{0.33} \mathrm{MnO}_{3} / \mathrm{LaAlO}_{3}$ heteroepitaxial system. It is found that while the position of the film-substrate interface is blurred by cation intermixing, oxygen vacancies selectively accumulate at the tensile region of the dislocation strain field. Such accumulation of vacancies is accompanied by the reduction of manganese 
cations in the same area, inducing chemical expansion effects which partly accommodate the dislocation strain. The formation of oxygen vacancies is only partially electrically compensated and results in a positive net charge $q \sim+0.3 \pm 0.1$ localized in the tensile region of the dislocation, while the compressive region remains neutral. Our results highlight a prototypical core model for perovskite based heteroepitaxial systems and offer insights for predictive manipulation of misfit dislocation properties.

\section{Introduction}

Dislocation cores can be considered as distinct filamentary-like nanophases [1,2] ubiquitous in crystalline solids and as such may severely impact their functional properties. Dislocations were conceptually conceived eight decades ago [3], even before they were observed in the transmission electron microscope [4]. Knowledge of their chemical and structural properties has attained a high level of maturity in metals [5-7]; however, their behavior in oxides still pose fundamental issues. The main differences between dislocations in metals and in oxides stem from the strong ionic character of the latter, making electrostatic interactions between their constituents crucial in determining the core structure and the distribution of dopants in the associated strain field, while in metals such effects are negligible [8,9]. Particularly in the case of oxides, oxygen vacancies play an important role in the development of electrostatic interactions and their formation has been recently the subject of several investigations. On the one hand, atomistic simulations show that oxygen vacancies accumulate at the undercoordinated structure of the dislocation core [10-13]. Similar studies also indicate a significant decrease/increase of the vacancy formation energy at the tensile/compressive strained zones neighboring the subnanometer core [11,12], or a decrease in both zones [14]. The coupling between strain and point defect formation is favored by volume changes that typically accompany the latter, and may lead to non-linear effects deviating from the elastic behavior of the bulk equilibrium phase $[15,16]$. In contrast with the formation of Cottrell 
atmospheres in metals, which can be modeled by the elastic interaction between a "misfitting" solute atom and a dislocation [17], dopant segregation around dislocation cores in oxides is determined by a complex interplay between elastic and electrostatic effects [12].

To date, the effect of dislocations on the performance of a variety of functional oxides has been documented, including high-temperature superconductors [18], catalysts [19], photovoltaics [20], thermoelectrics [21], ferroelectrics [22] and multiferroics [23]. The recognition of this impact has motivated in recent years a strong interest on their complex core structures $[10,24,25]$ and particularly their ionic and electronic transport properties at the atomic level $[10,12,26]$. The amazing complexity of the structural and functional behavior that dislocations can exhibit in complex oxides is exemplified by the $\mathbf{b}=[010]$ edge dislocation in $\mathrm{SrTiO}_{3}$ (STO), which has been the object of renewed interest since redox-based resistive switching behavior associated to its core was reported [27]. According to high resolution transmission electron microscopy (HRTEM), high angle annular dark field scanning TEM (HAADF-STEM) imaging and electron energy loss spectroscopy (EELS) studies, its core is dissociated and deficient in strontium and oxygen [28-31]. Moreover, a deficit in Sr has been shown to eventually induce a local transition to a fcc edge-sharing $\mathrm{TiO}_{6}$ octahedral structure with a larger unit cell accommodating the tensile strain of the dislocation [29-31]. Atomistic simulations, on the other hand, show that the experimentally observed dissociated core structure is only reproduced after removing an entire oxygen column from the undissociated core $[10,32]$. This suggests that the transition between both configurations is primarily governed by oxygen stoichiometry and that the dissociated core corresponds to the thermodynamically stable oxygen deficient structure. Remarkably, the accumulation of oxygen vacancies at the subnanometer core also affects the defect chemistry of the neighboring material on length scales ranging from several tens to hundreds of nanometers through the formation of oxygen vacancy depleted space charge zones [10,11,32,33].

Analogously to the behavior displayed by ferroelastic or ferroelectric domain walls, in 
which the localized strain may produce a variety of states not observed in the bulk phase [34], or even catalyze the formation of new phases with distinct physical and chemical properties [35], dislocation cores hold strong promise as potential discrete functional elements in perovskite oxides. However, unlike domain walls, which commonly form highly periodic patterns [36,37], the topological arrangement of dislocations is more difficult to control. One strategy to promote dislocation self-organization is to confine them onto the same crystallographic plane, where lateral order results from elastic interactions between neighboring lines, as it occurs at mismatched epitaxial heterointerfaces [38]. Such MDs are located at the interface bridging two different compounds and therefore their structure and chemical composition is expected to combine both of them. Despite the fact that the chemical and structural properties of MDs are even less understood than those of dislocations occurring in bulk crystals, recent reports highlight the strong potential of buried, laterally ordered MD networks in creating a variety of periodic strain-induced functional patterns in oxide thin films [39-41]. For instance, the periodic strain field associated with MD networks has been proposed to induce interfacial modulations of charge density [39], and more recently reported to induce the formation ordered topographic nanostructures [40,41] and electrical conduction patterns [41] at free surfaces. These recent findings highlight the strong impact of MDs in a range of length scales, and motivate the analysis of the distinct chemical and structural states confined in their cores, here referred to as MD phase, in view of the development of dislocation based nanostructures.

Prompted by the strong interest of MDs as spontaneously organized functional elements, in this work we investigate the MD phase in the $\mathrm{La}_{0.7} \mathrm{Sr}_{0.3} \mathrm{MnO}_{3} / \mathrm{LaAlO}_{3}$ (LSMO/LAO) epitaxial system to unveil the complex interplay between strain, chemical composition, and electronic structure that governs its formation. We choose the compressive LAO substrate to induce the formation of MDs, because under a tensile strain (on STO 
which the localized strain may produce a variety of states not observed in the bulk phase [34], substrates) the LSMO film relaxes by an octahedral tilting mechanism without dislocation 
formation [42]. On the other hand, in the LSMO/LAO system, MD networks exhibit a high

degree of order at relatively small thicknesses [43]. Although chemical information from individual atomic columns in defect structures is commonly accessed by EELS [44,45], here we demonstrate the advantages of combining EELS, for electronic structure analysis, with energy dispersive X-ray (EDX) spectroscopy for elemental quantification, all at atomic resolution. On one hand, since electronic delocalization scales inversely to the ionization energy, the much better efficiency of EDX in collecting X-ray signals from deep inner-shell ionizations in the incoherent imaging mode, makes EDX images more readily interpretable $[46,47]$; on the other hand, the range of accessible elements by EDX is less restricted than in EELS as it allows elemental mapping over a wide range of ionization energies, making this technique particularly suited to the present work. However, recent work by Craven et al. suggests that EELS on edges up to $10 \mathrm{keV}$ loss may be possible with improved microscopespectrometer alignments [48]. Our results indicate that the cationic distribution around the MD core is not solely dictated by elastic interactions, as reported for low aliovalent dopant concentrations in other oxides such as $\mathrm{CeO}_{2}$ [12] or $\mathrm{YBa}_{2} \mathrm{Cu}_{3} \mathrm{O}_{7}$ [49], where larger cations are stabilized in the tensile regions of the dislocation strain field. The present results provide key elements for understanding the mechanisms governing the chemistry and electronic structure of MDs in complex oxides as a first step towards the design of laterally modulated functional heterointerfaces over a $20 \mathrm{~nm}$ length scale.

\section{Results and Discussion}

Figure 1a shows a cross section high resolution HAADF image, viewed along the [100] zone axis, revealing dislocations at the interface between the LSMO film and the LAO substrate. The bottom panel is a (010)-Fourier filtered image pointing out the positions of the MDs. The 
formation [42]. On the other hand, in the LSMO/LAO system, MD networks exhibit a high extra half plane resides in the substrate side to accommodate the $-2.3 \%$ biaxial compressive

mismatch. In the LSMO/LAO system, both crystal structures are polar, being described by the 
stacking of charged atomic layers along the [001] direction: $\mathrm{Mn}^{3.3+} \mathrm{O}^{2-}{ }_{2}(-$

$2 / 3 e) / \mathrm{La}^{3+}{ }_{0.7} \mathrm{Sr}^{2+} \mathrm{O}^{2-}(+2 / 3 e)$ and $\mathrm{Al}^{3+} \mathrm{O}^{2-}(-1 e) / \mathrm{La}^{3+} \mathrm{O}^{2-}(+1 e)$, where the net charge per unit 0.3

2

area in the atomic planes is given in parenthesis and $e$ is the electron charge. The (001)

surface of the LAO substrate is known to be reconstructed to screen the diverging electrostatic potential arising from the stacking of dipole moments perpendicular to the surface [50-52]. Regardless of which type of (001) surface reconstruction is adopted by the LAO substrate, the LSMO/LAO interface introduces a polar discontinuity that can be screened either by moving electronic charges or ions [53]. Elemental concentration profiles determined by EDX (see Figure S1), obtained along the growth direction at positions in between MDs, indicate cation intermixing between the film and the substrate, and therefore interfacial charges are very likely avoided by chemical grading [53]. Figure S1 clearly shows that for B-site cations in the $\mathrm{ABO}_{3}$ perovskite (Mn, $\mathrm{Al}$ ) the diffusion length extends up to 3.6nm across the glide plane, whereas it is shorter, 2.1nm, for A-site cations ( $\mathrm{La}, \mathrm{Sr})$. A similar asymmetry between the extent of A- and B-site intermixing has been reported for other perovskite epitaxial films [54]. Since this chemical disorder precludes a precise identification of the interface plane, we use hereafter the position of the glide plane of the MDs as a reference. In addition, the oxygen concentration profile shows a slight constant depression, 3at\%, on the film side, indicating a background level of oxygen vacancies, $\mathrm{V}_{\mathrm{O}}$ (expressed using the Kröger-Vink notation [55]), throughout the film volume.

An atomic column resolution HAADF image of a dislocation core is presented in Figure 1b. Since this is an incoherent image in which the brightness of atomic columns is 
proportional to the atomic number, Z, brighter columns correspond to the $\mathrm{La} / \mathrm{Sr}$ (film) or $\mathrm{La}$ (substrate) atom columns. A Burgers circuit is drawn in the image, yielding a Burgers vector $\mathbf{b}=a[010]$ parallel to the interface. Therefore, the glide plane of the MDs, i.e. the plane containing the dislocation line and its Burgers vector, coincides with the interface plane. Note that in the perfect $\mathbf{b}=a[010]$ dislocation, the extra half plane is composed by an A-site plane 
plus a B-site/O plane. Since B-site/O columns are hardly visible owing to their lower atomic number, at first sight the image suggests an unrealistic perfect dislocation with a single extra half-plane of A-site columns in the substrate side. However, careful analysis of the image reveals that the core is dissociated in two partials corresponding to the two (hardly visible) half extra planes of B-site/O columns running along the two vertical arrows shown in the image. Further confidence for this core structure comes from the analysis of HRTEM images, as discussed in the next paragraph (Figure 2a). Thus, the MD is dissociated according to $\mathbf{b}=$ $a / 2[010]+a / 2[010]$ (here $a$ is the LAO lattice parameter), and the extended core contains an antiphase boundary on the glide plane between A-site columns (below the glide plane) and Bsite columns (above the glide plane). The vertical plane containing the antiphase boundary, passing between the two partials, will be hereafter referred to as axial plane. Interestingly, inspection of Figure 1b reveals that the two B-site atomic columns in the axial plane next to the antiphase contact, on the film side, exhibit higher brightness than the adjacent $\mathrm{Mn} / \mathrm{O}$ columns. These two positions are clearly identified in the vertical contrast profile shown in Figure 1c. The increased intensity at those two B-site columns points to their partial occupancy by heavier $\mathrm{La}^{3+}$ or $\mathrm{Sr}^{2+}$ cations, forming $\mathrm{La}_{\mathrm{Mn}}^{\mathrm{x}}$ or $\mathrm{Sr}_{\mathrm{Mn}}^{\prime}$ antisite defects, where $\mathrm{Mn}^{3+}$ is taken as the manganese reference state. Comparing the ionic radii of $\mathrm{La}^{3+}$ and $\mathrm{Sr}^{2+}$ in octahedral coordination, $1.032 \AA$ and $1.18 \AA$, respectively, with that of $\mathrm{Mn}^{3+}, 0.645 \AA$ [56], the driving force for the formation of such defects is considered to be the accommodation of the tensile strain above the glide plane. Similar antisite defects have been observed in an analogous misfit dislocation core in $\mathrm{BiFeO}_{3}$ [57]. An atomic core model derived from the present analysis is shown in Figure 1d. The composition of the glide plane is identified as Bsite/O. The reverse structure, namely that in which the two half extra planes are built by Asite columns, has also been identified in the course of this work. This configurations is thus 
similar to that previously identified in STO bicrystals $[10,28,29]$, and according to those 
studies, its occurrence can be tentatively considered as a fingerprint of oxygen

substoichiometry localized at the dislocation core.

Figure 2a is an HRTEM image corresponding to a dislocation core similar to that shown in Figure 1b. According to the multislice image simulations (defocus value $75 \mathrm{~nm}$, foil thickness $14.9 \mathrm{~nm}$ ) included as insets, the bright positions correspond to B-site/O columns, and therefore the image is complementary with the HAADF image shown in Figure 1b. The corresponding dilatation map, defined as $D_{x z}=\Delta V / V=\varepsilon_{X X}+\varepsilon_{z z}$, where $V$ is the volume, and $\varepsilon_{x x}$ and $\varepsilon_{z z}$ are the normal in-plane and perpendicular strain components, respectively, is depicted in Figure 2b. $D_{x z}$ represents the fractional change in volume that can be correlated with the size of cations redistributed around the MD core to accommodate the dislocation strain field. The map was obtained from Figure 2a by geometrical phase analysis [58], using the LAO substrate as a reference; therefore $D_{x z}$ values are somewhat exaggerated at the film side though it is difficult to quantify this effect due to the effect of cation intermixing on the lattice

parameters. Figure 2c shows the corresponding theoretical strain field obtained by adding the strain components of two 1/2[010] partial dislocations reproducing the configuration shown in Figure 1d. As a rough approximation, the calculation was performed using classical isotropic linear elasticity and therefore ignores the effects of the defect chemistry on the elastic behavior. The calculation also ignores the interface between the film and the substrate, and a Poisson's ration of 0.35 was used [41]. Note that owing to the observed cationic intermixing, a more realistic description should consider a compositional gradient instead of a sharp interface. Comparison between the experimental and theoretical maps reveals that while the latter exhibits a sharp boundary between the tensile and compressive regions at the glide plane, in the experimental map a positive dilatation region extents between the two partials towards 
studies, its occurrence can be tentatively considered as a fingerprint of oxygen the substrate. This anomaly will be addressed below.

The chemical composition around the dislocation core was investigated by atomic

resolution EDX mapping. Figure 3 shows the HAADF image of a dislocation core (a) along 
with its corresponding La- $\mathrm{L}_{\alpha}$ (b), Al- $\mathrm{K}_{\alpha}$ (c), Mn-K $\mathrm{K}_{\alpha}(\mathrm{d}), \mathrm{Sr}-\mathrm{K}_{\alpha}(\mathrm{e})$ and $\mathrm{O}-\mathrm{K}_{\alpha}$ (f) net count maps.

The maps show some fingerprints of the MD core on the elemental distribution, particularly visible for Sr, $\mathrm{Mn}$, and to a lower extent for $\mathrm{Al}$ and $\mathrm{O}$. There is a depletion of $\mathrm{Sr}, \mathrm{Al}$ and $\mathrm{O}$ ions around the core region, while there is a slight enrichment in Mn ions below the dislocation core.

Elemental concentrations were obtained by integrating net counts over two unit cell thick windows located above and below the glide plane, as indicated in Figure 3a. The integration generated a horizontal profile that was fit to a collection of Guassian peaks centered at equidistant positions at the corresponding ion sites, all with equal width and free intensities. The integrated areas of each Gaussian were then normalized to a reference value taken at a position sufficiently appart from the MD core to be considered as the bulk LSMO and LAO values. The conversion of X-ray net counts to atomic concentrations was performed by the Cliff-Lorimer method assuming the thin foil criterion [59]. The k-factors were obtained from a reference zone, considering a supercell $\left(\mathrm{La}_{0.7} \mathrm{Sr}_{0.3} \mathrm{MnO}_{3}\right)_{\mathrm{x}}\left(\mathrm{LaAlO}_{3}\right)_{\mathrm{y}}(\mathrm{x}=7$ and $\mathrm{y}=7)$.

The error bars in the concentration profiles were estimated from the scatter of results obtained

by varying $\mathrm{x}$ and $\mathrm{y}$ (further details on the procedure are given in the SI). Concentration profiles corresponding to above and below the glide plane are presented in Figure $\mathbf{3 g}$ and h, respectively. The position of the axial plane is indicated by arrows. The elemental concentration profiles are not strictly symmetric with respect to the axial plane very likely due to inhomogeneities in the strain field and some inaccuracies in the integration reflected by the error bars. It can be clearly seen that the distribution of elements differs markedly above and below the glide plane, in the tensile and compressive regions of the MD core, respectively. In the tensile region (Figure 3g), there is a clear enrichment in La accompanied by a depletion of Sr, although the maximum 2.5 at\% La enrichment is not fully compensated by the decrease 


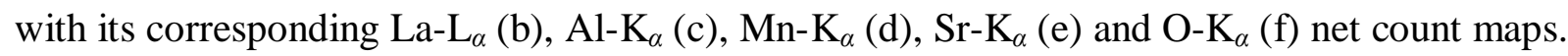
in Sr. This enrichment indicates that the increased brightness already observed at B-site

positions in Figure 1b, is very likely due to the formation of $\mathrm{La}_{\mathrm{Mn}}^{\mathrm{x}}$ antisite defects localized 
on the axial plane. On the other hand, the inbalance between La enrichment and Sr depletion

extends laterally a few unit cells at each side of the MD and therefore cannot be explained by the formation of LaMn antisite defects. We note, however, that a perfect balance (and site stoichiometry) is kept within the error bars. It is also worth noting that, taking into account the ionic radii of $\mathrm{La}^{3+}$ in cuboctahedral coordination, 1.36 $\AA$, and $\mathrm{Sr}^{2+}, 1.44 \AA$ [56], the depletion of larger Sr atoms goes against the accommodation of the tensile strain above the glide plane.

Figure 3h, on the other hand, shows that below the glide plane (compressive region), the La profile is $\sim 18$ at $\%$ and remains unperturbed by the presence of the $\mathrm{MD}$, while the $\mathrm{Sr}$ concentration still shows a slight depression below the core. The behavior of B-site cations shows different trends. Comparing Figure $3 g$ and h, one observes that above the glide plane they do not exhibit significant variations, while below it, the Mn concentration shows an increase below the position of the MD core, accompanied by a complementary depletion of $\mathrm{Al}$. The preferential substitution of $\mathrm{Al}^{3+}$ (ionic radius, $0.535 \AA$ ) by larger $\mathrm{Mn}^{3+}(0.645 \AA)$ [56] in the compressive region is again counterintuitive. However, this localized segregation of Mn nicely correlates with the anomalous dilatation observed in a similar dislocation, as shown in Figure 2b, suggesting that it might be favored by the specific core structure.

Comparing the oxygen concentration profiles obtained from the tensile and compressive regions of the MD, it becomes apparent that the glide plane marks the boundary between two well differentiated ionic landscapes. As commented above, vertical elemental concentration profiles obtained from an integration area located between MDs indicate that there is a background $V_{0} \ddot{c}$ concentration of about 3at\%. Comparing Figures $3 g$ and h, this background $\mathrm{V}^{\ominus}$ level is preserved in the first two unit cells above and below the glide plane, but is selectively increased in the tensile region around the MD core. This behavior strongly 
on the axial plane. On the other hand, the inbalance between La enrichment and Sr depletion suggests that the vacancy formation energy is further reduced under tensile strain, in

agreement with atomistic simulations of dislocations in $\mathrm{CeO}_{2}$ [12] and STO [11], and biaxially strained thin films of $\mathrm{CaMnO}_{3}[60,61]$. 
The electronic structure of the dislocation core and its vicinity was investigated by

analyzing the O-K and Mn-L energy-loss near-edge structure (ELNES). Since the strong strain field of the MD can affect the ELNES and these effects are not well understood, we paid special attention to the determination of the Mn oxidation state. Computer simulations of the $\mathrm{Mn}^{3+}-\mathrm{L}_{2,3}$ edge under compressive and tensile strain indicated a negligible effect on those spectral features sensitive to the oxidation state (see Figure S12). Here we report results obtained using the two most widely used techniques to determine the Mn oxidation state in manganites, namely measuring the red shift of the $\mathrm{Mn}-\mathrm{L}_{3}$ maximum and the ratio between the $\mathrm{L}_{3}$ and $\mathrm{L}_{2}$ intensities [62]. Figure 4 examines the evolution of the corresponding spectra along the interface normal, across the dislocation core. The spectra were extracted from unit cell areas, DF3 to DS3, as indicated in Figure 4a. In Figure 4b the dashed spectrum, F, corresponds to a location in the film far away from the dislocations and is included here for reference. Spectrum S corresponds to a position below the glide plane beyond which the Mn signal vanishes and is used as a reference for the LAO substrate. The glide plane is located between spectra DF1 and DS1.

The $\mathrm{O}-\mathrm{K}$ edge is interpreted in terms of the transition from $\mathrm{O} 1 \mathrm{~s}$ core levels to $\mathrm{O} 2 p$ states hybridized with transition metal states [63]. It typically features three peaks, labeled a, b and $\mathbf{c}$ in Figure 4b. Peak a, commonly referred to as prepeak, appears around $531 \mathrm{eV}$, and is attributed to manganese $3 d$ states. This peak is sensitive to the Mn $3 d$ band occupancy; thus, in the expected high-spin state, it is weakest for $\mathrm{Mn}^{3+}$ with its $3 d t_{2 g}$ spin-up levels fully occupied and one electron in the $e_{g}$ level, and becomes stronger as the concentration of $\mathrm{Mn}^{4+}$ increases. The second peak, b, found around $536 \mathrm{eV}$, is attributed to the hybridization with the A-site La $5 d$ or Sr $4 d$ bands. A noticeable broadening of peak $\mathbf{b}$ is observed as the A-site, occupied only by La cations in the substrate, becomes progressively shared with Sr cations in the film. The third peak, c, found around $543 \mathrm{eV}$, is related to the projected unoccupied 
The electronic structure of the dislocation core and its vicinity was investigated by oxygen $p$ states mixed with the B-site $4 s p$ band. As the glide plane is approached from the 
film side, the intensity of peak c vanishes and recovers again below the glide plane, which can

be tentatively attributed to the severe distortion of the $\mathrm{BO}_{6}$ octahedra near the $\mathrm{MD}$ core.

Figure $4 \mathrm{~b}$ shows that in all the spectra above the glide plane, along with that obtained from one unit cell just below it, the prepeak is hardly visible, much weaker than in the reference spectrum, F, pointing to a preferential reduction of the Mn cations along the axial plane of the MD.

The simultaneously acquired Mn- $\mathrm{L}_{2,3}$ edges, depicted in Figure 4c, on the other hand, show concomitant modifications of their near edge structures. This edge consists of two white lines, $\mathrm{L}_{2}$ and $\mathrm{L}_{3}$, attributed to the transition from the spin orbit split $2 p_{3 / 2}$ and $2 p_{1 / 2}$ levels to available states in the $3 d$ unoccupied band [63]. Variations in the $\mathrm{L}_{2} / \mathrm{L}_{3}$ intensity ratio or chemical shifts, are typical fingerprints of changes in the oxidation state [62]. In particular, Figure 4c shows a red shift and an increase of the $\mathrm{L}_{3} / \mathrm{L}_{2}$ ratio relative to the $\mathrm{F}$ spectrum in all the spectra taken above the glide plane and one unit cell below it. The variation of $L_{3}$ peak energy with the vertical position across the dislocation core is shown in Figure 4d. A red shift value of $-0.45 \mathrm{eV}$ is kept almost constant from DF3 to DS1, which assuming the nominal oxidation state +3.33 for the unperturbed LSMO film, indicates an oxidation state of $+3.03 \pm$ 0.03 in the dislocation core. Two unit cells below the glide plane the Mn oxidation state increases until its concentration vanishes. The increase of the $\mathrm{L}_{3} / \mathrm{L}_{2}$ ratio observed in Figure 4e supports a similar trend [63-65]. Taking the value obtained from spectrum $\mathrm{F}$ as a reference

for the nominal +3.33 value, we obtain an oxidation state of $+2.7 \pm 0.2$ for unit cell areas DF3 down to DS1, slightly smaller than that obtained by the chemical shift method. We therefore adopt an oxidation state of +3 for Mn cations located in the neighborhood of the MD core, as a compromise between the values obtained by both methods.

Figure 5 shows the lateral variation of the EEL O-K (a) and Mn- $\mathrm{L}_{2,3}$ (b) spectra along 
film side, the intensity of peak $\mathbf{c}$ vanishes and recovers again below the glide plane, which can the glide plane, as well as a HAADF image indicating the positions from which the spectra were extracted. The prepeak intensity in the O-K edge spectrum is weakest at the MD core 
(position GP0 in Figure 5c). The intensity of peak c also decreases at this position in agreement with the behavior observed in the vertical profiles shown in Figure 4. As observed in Figure 5b, the trend observed in the $\mathrm{Mn}-\mathrm{L}_{2,3}$ spectra is consistent with the behavior of the O-K edge prepeak intensity. The $\mathrm{L}_{3}$ peak is shifted towards lower energies at the MD core,

indicating that the fluctuation in the Mn oxidation state caused by the dislocation is confined within a narrow region of about 5 unit cells. Beyond this distance, the Mn oxidation state adopts the value determined at the glide plane in regions far from the MDs, $+3.20 \pm 0.03$. According to these results, the electrical charge per unit cell at the MD core was estimated assuming a Mn oxidation state of +3 and the cation stoichiometry derived from EDX analyses. Results obtained above and below the glide plane are shown in Figure 6a and b, respectively. Notably, it can be observed that the behavior is drastically different under tensile (b) and compressive (a) scenarios. In the former case, a positive charge of $q$ $+0.3 \pm 0.1$ decorates the MD core within a lateral range of 4 unit cells and decays with distance from the dislocation, while in the latter, charge neutrality is kept through the position of the MD. Therefore, the charge enrichment appears to be spatially correlated with the $\mathrm{V}_{0}^{*}$

concentration. This positive charge on the tensile side will have a compensating charge in the surrounding material, but unfortunately our work does not provide sufficient evidence to determine the mechanism of charge compensation. The observation of positively charged MDs draws a scenario opposed to that reported for MDs in a similar manganite based heteroepitaxial system, $\mathrm{Nd}_{0.35} \mathrm{Sr}_{0.65} \mathrm{MnO}_{3} / \mathrm{STO}$ [39]. The mechanism proposed by those authors considers the reorganization of 2D pristine interfacial charges, present prior to the formation of MDs, into 1D chains along the dislocation strain fields when MDs are introduced. 
(position GP0 in Figure 5c). The intensity of peak c also decreases at this position in In that case, however, no experimental attempt to determine the oxygen concentration was

performed and unit cell charges were estimated neglecting any possible effect of oxygen vacancies. 
An attempt to describe the basic mechanisms involved in the distribution of cations and oxygen vacancies around the MD core is schematically summarized in Figure 7. As indicated on the schematic, contrary to what would be expected considering only atomic size effects, Sr cations, larger than La, exhibit a slight depletion while the La/Sr ratio increases in the tensile zone. This suggests a counterbalancing volume effect of the formation of $\mathrm{La}_{\mathrm{Mn}}^{\mathrm{x}}$

antisite defects, which according to the Shannon ionic radius of $\mathrm{La}^{3+}$ and $\mathrm{Mn}^{3+}$ in octahedral coordination, $1.032 \AA$ and $0.645 \AA$, respectively [56], yield a huge localized positive dilatational strain of $60 \%$. However, the strain field associated with a point strain source is known to induce a shear without dilatation in the neighboring matrix as well as to decay as $1 / r^{3}$ with distance, $r$, from it [66]. Therefore, the strain accommodating effect of point defects

is very local. In this sense, the observed oxygen depletion over the tensile zone suggests a dilatational effect of oxygen vacancies.

The radius of an oxygen vacancy in the perovskite structure can be considered similar or slightly smaller than the radius of an oxide ion [67] and therefore the accumulation of $\mathrm{V}^{* *} \mathrm{~s}$ are expected to have only a small or negligible effect on the accommodation of the MD strain.

Nevertheless, the spatial correlation between the concentration of $V_{0}$ and $M n$ suggests a strain compensating effect associated with the reduction of Mn cations. The redox reaction can be expressed as: 


$$
\begin{array}{ccc}
\mathrm{x} & \mathrm{x} & 1 \\
\mathrm{O}_{\mathrm{O}}+2 \mathrm{Mn}_{\mathrm{Mn}} \leftrightarrow \mathrm{V}_{\mathrm{O}}+2 \mathrm{Mn}_{\mathrm{Mn}}+{ }_{2} \mathrm{O}_{2} \uparrow
\end{array}
$$

where $\mathrm{Mn}^{3+}$ is used as the reference state for the host B-site. According to Equation (1), each $\mathrm{V}^{*}$ leaves two free carriers [68] that are transferred to the Mn $d$ band (though at high vacancy 0

concentrations this number can be less [69]), thus in principle reducing two neighboring $\mathrm{Mn}^{4+}$ cations. Comparing the ionic radius of the average $\mathrm{Mn}^{3+}{ }_{0.7} \mathrm{Mn}_{0.3}^{4+}$ cation, $0.610 \AA$, with that of 
$\mathrm{Mn}^{3+}, 0.645 \AA$, a chemical expansion of $\sim 6 \%$ associated with the formation of oxygen vacancies can be estimated. We note, however, that considering the observed substitution level of $\mathrm{Mn}^{3+}$ by $\mathrm{Al}^{3+}$ (octahedral radius, $0.535 \AA$ [56]) of 1/4, see Figure 3g, the radius of the average B-site cation becomes $0.617 \AA$, close to the bulk value $0.610 \AA$. Hence, within experimental errors, there are no significant differences between the ionic radii of the average B-site cations located in the tensile region and those located in the bulk film far from the influence of the MDs. This suggests that despite the observed cation intermixing, most of the MD strain is accommodated by elastic distortion of interatomic bonds. Strikingly, the slight depletion of Sr, leading to a decrease in size of the average A-site cations in the tensile zone, is not compensated by any significant dilatation of the B-sites. Therefore, we can conclude that the unique significant chemical expansion effect contributing to the relaxation of the tensile strain above the glide plane of the MD, comes from the formation of La Mn antisite defects on the axial plane, which as commented above, have a very local compensation effect.

Interestingly, the results of the present study are in contrast to atomistic simulations of the distribution of trivalent dopants around an edge dislocation in ceria [12]. In that case, the driving force governing the distribution of dopants is the minimization of the elastic energy, i.e., larger dopants accumulate in the tensile zone whilst smaller ones replace $\mathrm{Ce}^{4+}$ in the compressive zone. Those authors also conclude that the spatial distribution of $V_{0}$ is controlled by electrostatic interactions: positively charged oxygen vacancies are attracted by negatively charged trivalent dopants regardless of whether they are located in the tensile or compressive regions of the dislocation. Following a similar mechanism, in our case one would expect the association of positively charged oxygen vacancies, $\mathrm{V}_{0}$, accumulated in the tensile zone, with 
negatively charged $\mathrm{Sr}_{\mathrm{La}}^{\prime}$ species, in contrast with the decrease of the Sr concentration

observed in Figure 3g,h. In the present case, however, the doping level of divalent Sr cations, $33 \%$, is much higher and the chemical behavior of the system very likely cannot be properly described by individual point defect interactions. Taking into account the LSMO 
stoichiometry, $\mathrm{La}^{3+} \mathrm{Sr}^{2+} \mathrm{Mn}^{3+} \mathrm{Mn}^{4+} \mathrm{O}$, and neglecting $\mathrm{Al}$ interdiffusion, charge $1-\mathrm{x} \quad \mathrm{x} \quad 1-\mathrm{y} \quad \mathrm{y} \quad 3-\delta$

neutrality imposes $\delta=(\mathrm{x}-\mathrm{y}) / 2$. Since, on the basis of the present EELS analysis, both, Mn cations located in the neighborhood of the $\mathrm{MD}$ core and $\mathrm{Al}$, are in an oxidation state of +3 , the charge neutrality condition becomes $\delta=\mathrm{x} / 2$, which in Kröger-Vink notation reads as

$\otimes \mathrm{Sr}_{\mathrm{La}} \curvearrowright 2\left[\mathrm{~V}_{\mathrm{O}}\right]$. According to this relation, the electrostatic energy arising from the charge unbalance induced by the formation of oxygen vacancies could be canceled by enrichment in Sr over the tensile zone, contrary to the observed depletion (see Figure 7). Moreover, recalling that Sr cations are larger than La ones, such enrichment would simultaneously accommodate the tensile strain above the glide plane. On the other hand, charge compensation could be also favored by the formation of negatively charged cationic vacancies. Nevertheless, the cationic concentrations shown in Figure 3g,h, do not provide conclusive evidences for the occurrence of significant amounts of vacancies neither in the A nor the B sites. The observed behavior in fact points to a complex energy balance between elastic and electrostatic effects that cannot be completely captured from the present analysis.

\section{Conclusion}

The present work demonstrates that the detailed atomic and chemical structure of misfit dislocations in the complex oxide heterostructure LSMO/LAO can be quantitatively accessed by a combination of transmission electron microscopy imaging (HRTEM, HR-HAADF) and spectroscopic (EDX, EELS) techniques. The atomic structure shows a dissociation scheme 
similar to that found in oxygen deficient dislocation cores in STO $[10,28,29]$, suggesting that it may be considered as a model scheme for other perovskite on perovskite epitaxial systems. Here we provide experimental evidence of the selective formation of oxygen vacancies in the tensile zone of the dislocation, on the film side. As a result, a positive charge enrichment occurs at the tensile zone while the compressive one remains neutral. The dislocation glide plane thus defines a selective boundary regarding the distribution of oxygen vacancies, while 
allowing intermixing between the film and the substrate within a few unit cells. Oxygen vacancies, in turn, induce the reduction of neighboring Mn cations to $\mathrm{Mn}^{3+}$ and generate electrostatic interactions that ultimately govern the cationic distribution around the dislocation core. Notably, on the basis of averaged ionic radii, the resulting compositional modification does not directly correlate with the dislocation strain field, thus pointing to a complex balance between point defect formation, electrostatic and elastic energies. The present results provide essential insights into the complex chemical behavior of misfit dislocations formed at the interface between two multinary oxides.

\section{Experimental Section}

Films: High quality films with (100) orientation and thicknesses of 3.5 and 6nm, were epitaxially grown under a biaxial compressive strain of $-2.3 \%$ on LAO substrates by magnetron sputtering as reported elsewhere [70].

Transmission Electron Microscopy: Thin foil specimens were prepared by conventional cutting-gluing-grinding procedures, followed by Ar milling at a grazing incidence down to perforation. Cross-section atomic resolution images (HRTEM) were obtained at 200kV using the field-emission gun FEI Tecnai F20 S/TEM. Scanning transmission electron microscopy (STEM) combined with EEL spectrum imaging (SI) and energy dispersive x-ray (EDX) spectral mapping of cross-section samples, were performed in the image-corrected FEI $\operatorname{Titan}^{3 T M}$ G2 60-300 operating at 300kV, equipped with a Super-X four quadrant detector and

a Gatan Quantum dual EELS spectrometer. STEM images were acquired with a convergence angle of 10 mrad and an annular detector inner radius of $138 \mathrm{mrad}$. The detailed procedure 
employed for EDX data analysis is explained in the SI. The dual EELS-SI data were acquired using a 22 mrad collection angle. The EELS spectrometer was vertically binned by 130x to improve the readout speed. The zero-loss and core-loss, from $500 \mathrm{eV}$ to $900 \mathrm{eV}$, were acquired for every pixel compensating for the intensity difference between them. The core-loss 
acquisition time per pixel was $0.5 \mathrm{~s}$ and the low-loss spectrum was $0.001 \mathrm{~s}$. A dispersion of

$0.25 \mathrm{eV}$ per channel was used. Spatial drift corrections were applied in order to minimize drift. The energy resolution measured as the full width at half-maximum of the zero-loss peak was $\sim 1.75 \mathrm{eV}$. The zero loss was used to correct the energy shift of core loss. The principal components analysis (PCA) provided by Lucas et al. [71] for Digital Micrograph was used to remove random noise from the EEL spectrum. The power law function was used before each energy peak to remove the background, then deconvolution was performed using the Fourier Ratio method. The Mn- $\mathrm{L}_{2,3}$ ratio was measured following the procedure described by Varela et al. [61]. High angle annular dark field images (HAADF) were obtained in a probe-corrected $\operatorname{Titan}^{3 \mathrm{TM}} 80-300$ operating at $300 \mathrm{kV}$. The images of cross-section samples were acquired with a probe convergence angle of 10 mrad and an annular detector inner radius of 69 mrad. Image processing and simulation: HRTEM images were simulated by the multislice method using the jems software package [72]. Strain maps were computed from HRTEM images by geometrical phase analysis, GPA [58].

Simulation of EELS spectra: Atomic multiplet calculations were performed to simulate the $\mathrm{Mn}-\mathrm{L}_{2,3}$ edge under compressive and tensile uniaxial strain, using the CTM4XAS program [73]. Details on the simulations are given in the SI.

\section{Supporting Information}

Supporting Information is available from the Wiley Online Library or from the author. 
acquisition time per pixel was $0.5 \mathrm{~s}$ and the low-loss spectrum was $0.001 \mathrm{~s}$. A dispersion of

\section{Acknowledgements}

We thank Dr. Belén Ballesteros for her assistance in TEM experiments with the FEI Tecnai

F20 S/TEM microscope. This research was funded by the Spanish MINECO (projects:

MAT2011-29081-C02, MAT2012-33207, and MAT2013-47869-C4-1-P, Consolider-Ingenio

CSD2008-00023) and the European Union Horizon 2020 research and innovation programme 
under the Marie Sklodowska-Curie Grant Agreement No. 645658. ICN2 is supported by the Severo Ochoa program from Spanish MINECO (Grant No. SEV-2013-0295) and by the CERCA Programme (Generalitat de Catalunya). We also acknowledge financial aid from the Generalitat de Catalunya (2014 SGR 501 and 2014 SGR 1216). N.B. thanks the Spanish MINECO for the financial support through the FPI program. Z.K. is grateful for the support from the Ministry of Education, Science, and Technological Development of the Republic of Serbia through Project No. III45018. BDE and DWM acknowledge support from the Center for Emergent Materials at the Ohio State University, a National Science Foundation Materials Research Science and Engineering Center (Grant No. DMR-1420451). The ICN2 is funded by the CERCA programme / Generalitat de Catalunya, and supported by the Severo Ochoa programme of the Spanish Ministry of Economy, Industry and Competitiveness (MINECO, grant no. SEV-2013-0295).

References

[1] M. Kuzmina, M. Herbig, D. Ponge, S. Sandlöbes, D. Raabe, Science 2015, 349, 1080-

1083.

[2] M. E. Glicksman, C. L. Vold, Surf. Sci. 1972 31, 50-57.

[3] G. I. Taylor, Proc. R. Soc. Lond. A, 1934, 145, 362-387.

[4] P. B. Hirsch, R. W. Horne, M. J. Whelan, Phil. Mag. 1956, 1, 677-684.

[5] J. Huang, M. Meyer, V. Pontiki, Phys. Rev. Lett. 1989, 63, 628-631.

[6] L. Y. Chen, Mo-rigen He, J. Shin, G. Richter, D. S. Gianola, Nat. Mater. 2015, 14, 707- 
under the Marie Sklodowska-Curie Grant Agreement No. 645658. ICN2 is supported by the 713.

[7] M. Legros, G. Dehm, E. Arzt, T. J. Balk, Science 2008, 319, 1646-1649. 
[8] R. Smoluchowski, J. Phys. Colloq. 1966, 27 (C3), C3-3-C3-11.

[9] D. Hull, D. J. Bacon, Introduction to Dislocations, Elsevier, 2001, Oxford, UK.

[10] V. Metlenko, A. H. H. Ramadan, F. Gunkel, H. Du, H. Schraknepper, S. HoffmannEifert, R. Dittmann, R. Waser, R. A. De Souza, Nanoscale 2014, 6, 12864-12876.

[11] D. Marrocchelli, L. Sun, B. Yildiz, J. Am. Chem. Soc. 2015, 137, 4735-4748.

[12] L. Sun, D. Marrocchelli, B. Yildiz, Nat. Commun. 2015, 6, 6294.

[13] A. H. H. Ramadan, R. A. De Souza, Acta Mater. 2016, 118, 286-295.

[14] S.-Y. Choi, S.-D. Kim, M. Choi, H.-S. Lee, J. Ryu, N. Shibata, T. Mizoguchi, E. Tochigi, T. Yamamoto, S.-J. L. Kang, Y. Ikuhara, Nano Lett. 2015, 15, 4129-4134.

[15] M. Greenberg, E. Wachtel, I. Lubomirsky, J. Fleig, J. Maier. Adv. Funct. Mater. 2006, 16, 48-52.

[16] A. Kossoy, Y. Feldman, E. Wachtel, I. Lubomirsky, J. Maier. Adv. Funct. Mater. 2006,

$17,2393-2398$.

[17] A. H. Cottrell, B. A. Bilby, Proc. Phys. Soc. A 1949, 62, 49-62; L. V. Meisel, J. Appl. Phys. 1967, 38, 4780-4784.

[18] F. Sandiumenge, T. Puig, J. Rabier, J. Plain, and X. Obradors, Adv. Mater. 2000, 12, 
[8] R. Smoluchowski, J. Phys. Colloq. 1966, 27 (C3), C3-3-C3-11.

[19] A. Trovarelli, P. Fornasiero, Catalysis by Ceria and Related Materials, 2nd ed. Imperial College Press, 201, London.

[20] H. J. J. Snaith, Phys. Chem. Lett. 2013, 4, 3623-3630.

[21] S. B. Riffat, X. Ma, Appl. Therm. Eng. 2003, 23, 913-935.

[22] M.-W. Chu, I. Szafraniak, R. Scholz, C. Harnagea, D. Hesse, M. Alexe, U. Gösele, Nat. Mater. 2004, 3, 87-90.

[23] R. Ramesh, N. A. Spaldin, Nat. Mater. 2007, 6, 21-29.

[24] Z. Wang, M. Saito, K. P. McKenna, Y. Ikuhara, Nat. Commun. 2014, 5, 3239. 
[25] P. P. Dholabhai, G. Pilania, J. A. Aguiar, A. Misra, B. P. Uberuaga, Nat. Commun. 2014, $5,5043$.

[26] K. P. McKenna, J. Am. Chem. Soc. 2013, 135, 18859- 18865.

[27] K. Szot, W. Speier, G. Bihlmayer, R. Wasser, Nat. Mater. 2006, 5, 312-320.

[28] Z. Zhang, W. Sigle, M. Rühle, Phys. Rev. B, 2002, 66, 094108.

[29] H. Du, C.-L. Jia, L. Houben, V. Metlenko, R. A. De Souza, R. Waser, J. Mayer, Acta

Mater. 2015, 89, 344-350.

[30] J. P. Buban, M. Chi, D.J. Masiel, J.P. Bradley, B. Jiang, H. Stahlberg, N. D. Browning, J. Mater. Res. 2009, 24, 2191-2199.

[31] K. Takehara, Y. Sato, T. Tohei, N. Shibata, Y. Ikuhara, J. Mater. Sci. 2014, 49, 39623969.

[32] S. P.Waldow, R. A. De Souza, ACS Appl. Mater. Interfaces 2016, 8, 12246-12256.

[33] K. K. Adepalli, J. Yang, J.Maier, H.L. Tuller, B. Yildiz, Adv. Funct. Mater. 2017, 27, 1700243.

[34] G. Catalan, J. Seidel, R. Ramesh, J. F. Scott, Revs. Mod. Phys. 2012, 84, 119-156. 
[25] P. P. Dholabhai, G. Pilania, J. A. Aguiar, A. Misra, B. P. Uberuaga, Nat. Commun. 2014,

[35] S. Farokhipoor, C. Magén, S. Venkatesan, J. Íñiguez, C. J. M. Daumont, D. Rubí, E. Snoeck, M. Mostovoy, C. de Graaf, A. Müller, M. Döblinger, C. Scheu, B. Noheda, Nature 2014, 515, 379-383.

[36] N. Farag, M. Bobeth, W. Pompe, A. E. Romanov, J. S. Speck, J. Appl. Phys. 2005, 97, 113516.

[37] J. Santiso, Ll. Balcells, Z. Konstantinovic, J. Roqueta, P. Ferrer, A. Pomar, B. Martinez, F. Sandiumenge, CrystEngComm. 2013, 15, 3908-3918.

[38] S. C. Jain, A. H. Harker, R. A. Cowley, Phil. Mag. A, 1997, 6, 1461-1515.

[39] C.-P. Chang, M.-W. Chu, H. T. Jeng, S.-L. Cheng, J. G. Lin, J.-R. Yang, and C. H. Chen, Nat. Commun. 2014, 5, 3522. 
[40] S. Pandya, A. R. Damodaran, R. Xu, S.-L. Hsu, J. C. Agar, and L. W. Martin, Sci. Rep. 2016, 6, 26075.

[41] F. Sandiumenge, N. Bagués, J. Santiso, M. Paradinas, A. Pomar, Z. Konstantinovic, C. Ocal, Ll. Balcells, M.-J. Casanove, B. Martínez, Adv. Mater. Interfaces 2016, 3, 1600106.

[42] F. Sandiumenge, J. Santiso, Ll. Balcells, Z. Konstantinovic, J. Roqueta, A. Pomar, J. P. Espinós, B. Martínez, Phys. Rev. Lett. 2013, 110, 107206.

[43] J. Santiso, J. Roqueta, N. Bagués, C. Frontera, Z. Konstantinovic, Q. Lu, B. Yildiz, B. Martínez, A. Pomar, Ll. Balcells, F. Sandiumenge, ACS Appl. Mater. Interfaces 2016, 8, 16823-16832.

[44] G. A. Botton, MRS Bulletin 2012, 37, 21-28.

[45] M. Varela, J. Gazquez, S. J. Pennycook, MRS Bulletin, 2012, 37, 29-35.

[46] M. -W. Chu, S. C. Liou, C. -P. Chang, F. -S. Choa, C. H. Chen, Phys. Rev. Lett.. 2010, 104, 196101.

[47] A. J. D'Alfonso, B. Freitag, D. Klenov, L. J. Allen, Phys. Rev. B. 2010, 81, 100101(R).

[48] A. J. Craven, H. Sawada, S. McFadzean, I. MacLaren, Ultramicroscopy 2017, 180, 66-80.

[49] R. F. Klie, J. P. Buban, M. Varela, A. Franceschetti, C. Jooss, Y. Zhu, N. D. Browning, S. T. Pantelides, S. J. Pennycook, Nature 2005, 435, 475-478.

[50] A. J. H. van der Torren, S. J. van der Molen, J. Aarts, Phys. Rev. B 2015, 91, 245426. 
[40] S. Pandya, A. R. Damodaran, R. Xu, S.-L. Hsu, J. C. Agar, and L. W. Martin, Sci. Rep.

[51] C. H. Lanier, J. M. Rondinelli, B. Deng, R. Kilaas, K. R. Poeppelmeier, L. D. Marks,

Phys Rev. Lett. 2007, 98, 086102.

[52] H. Seo, A. A. Demkov, Phys. Rev. B 2011, 84, 045440

[53] N. Nakagawa, H. Y. Hwang, D. A. Muller, Nat. Mater. 2006, 5, 204-209.

[54] C.-P. Chang, J. G. Lin, H. T. Jeng, S.-L. Cheng, W. F. Pong, Y. C. Shao, Y. Y. Chin, H.J. Lin, C. W. Chen, J.-R. Yang, C. H. Chen, M.-W. Chu, Phys. Rev. B 2013, 87, 075129.

[55] F. A. Kröger, H. J. Vink, in: F. Seitz, D. Turnball (Eds.), Solid State Physics, 1956, p.

307. 
[56] R. D. Shannon, Acta Cryst. A 1976, 32, 751-767.

[57] A. Lubk, M. D. Rossell, J. Seidel, Y. H. Chu, R. Ramesh, M. J. Hÿtch, E. Snoeck, Nano. Lett. 2013, 13, 1410-1415.

[58] M. J. Hÿtch, Microsc. Microanal. Microstruct. 1997, 8, 41-57.

[59] G. Cliff, G.W. Lorimer, Journal of Microscopy, 1975, 103, 203-207.

[60] U. Aschauer, N. A. Spaldin, Appl. Phys. Lett. 2016, 109, 031901.

[61] U. Aschauer, R. Pfenninger, S. M. Selbach, T. Grande, and N. A. Spaldin, Phys. Rev. B 2013, 88, 054111.

[62] M. Varela, M. P. Oxley, W. Luo, J. Tao, M. Watanabe, A. R. Lupini, S. T. Pantelides, S. J. Pennycook, Phys. Rev. B 2009, 79, 085117.

[63] H. Kurata, C. Colliex, Phys. Rev. B 1993, 48, 2102-2108.

[64] T. Sparrow, B. Williams, C. Rao, J. Thomas, Chem. Phys. Lett. 1984, 108, 547-550.

[65] W. G. Waddington, P. Rez, I. P. Grant, C. J. Humphreys, Phys. Rev. B 1986, 34, 14671473.

[66] N. F. Mott, F. R. N. Nabarro, Proc. Phys. Soc. 1940, 52, 86-89.

[67] D. Marrocchelli, N. H. Perry, S. R. Bishop, Phys. Chem. Chem. Phys. 2015, 17, 10028- 
[56] R. D. Shannon, Acta Cryst. A 1976, 32, 751-767.

10039.

[68] H. Yamada, G. R. Miller, J. Solid State Chem. 1973, 6, 169-177.

[69] W. Gong, H. Yun, Y. B. Ning, J. E. Greedan, W. R. Datars, C. V. Stager, J. Solid State

Chem. 1991, 90, 320-330.

[70] Z. Konstantinovic, J. Santiso, D. Colson, A. Forget, Ll. Balcells, B. Martínez, J. Appl. Phys. 2009, 105, 063919.

[71] G. Lucas, P. Burdet, M. Cantoni, C. Hebert, Micron 2013, 52-53, 49-56.

[72] P. Stadelmann, Ultramicroscopy 1987, 21, 131-145.

[73] E. Stavitski, F. M. F. de Groot, Micron 2010, 41, 687-694. 

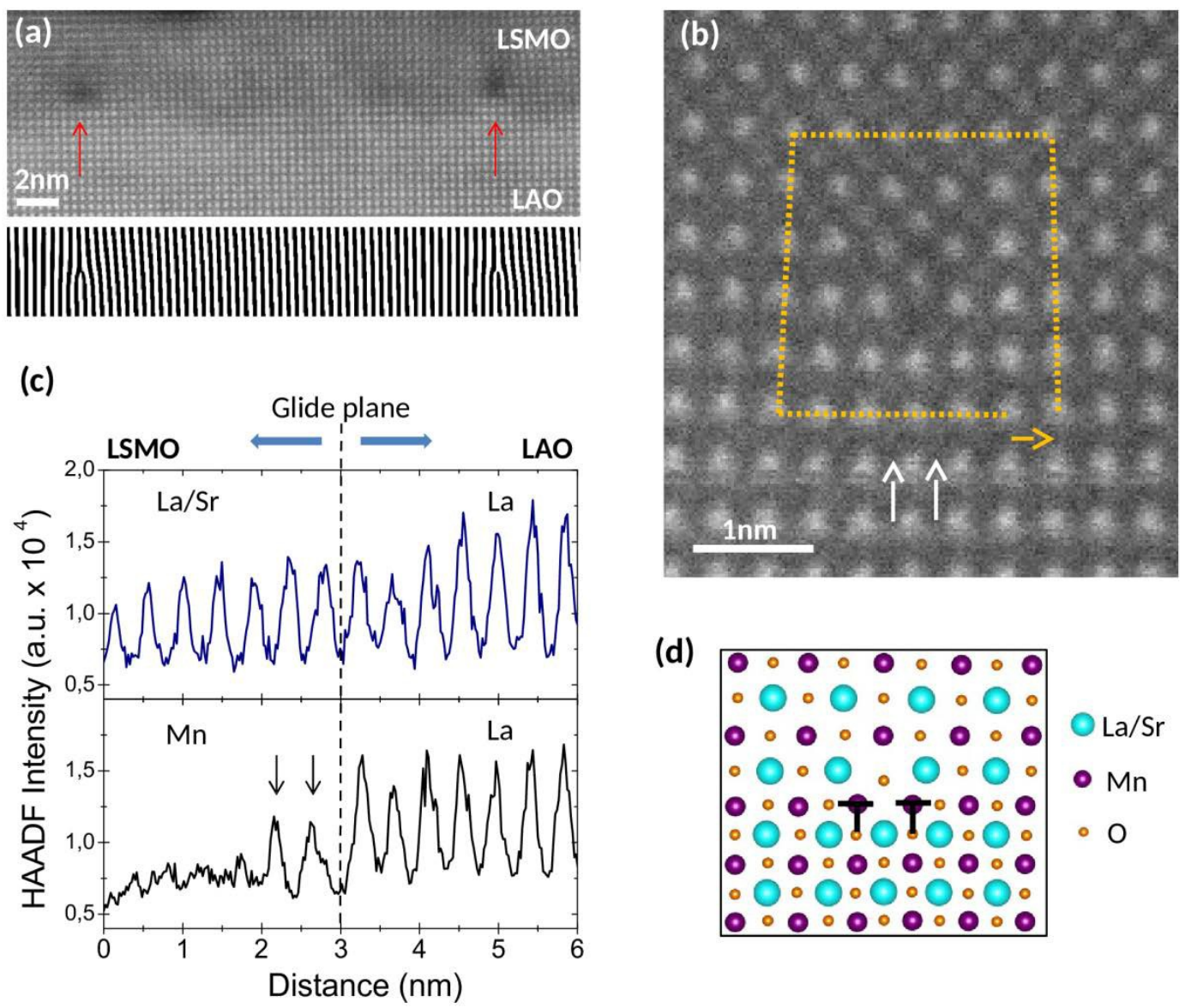

(d)

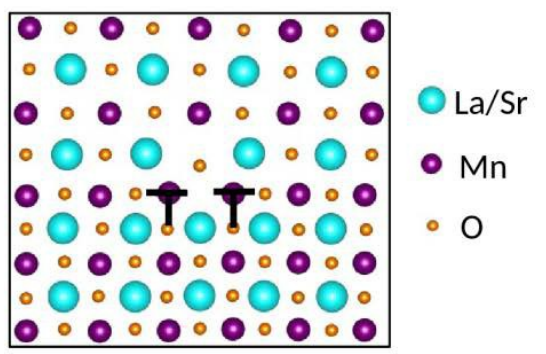

Figure 1. Misfit dislocations in partially relaxed LSMO/LAO thin films. (a) Atomic resolution cross-sectional HAADF image, viewed along the [100] zone axis, of a $6 \mathrm{~nm}$ thick LSMO/LAO film. Vertical arrows indicate the position of misfit dislocations, seen as dark contrast. Bottom panel: Fourier filtered image using the (010) frequency to reveal the dislocations. (b) Atomic resolution cross-sectional HAADF image, viewed along the [100] zone axis, of a misfit dislocation. The two arrows indicate the position of the two extra B- 
site/O (nominally Al/O) half planes of the dissociated core. These planes are hardly visible in the HAADF image due to their lower atomic number. Dotted lines draw a Burgers circuit, with the yellow arrow signifying the Burgers vector $\mathbf{b}=a[010]$. (c) Vertical intensity profiles taken along the axial plane containing the antiphase boundary (black) and an adjacent A-site (blue) atomic plane running through the dislocation core. The vertical line marks the position of the glide plane. The two arrows indicate the two positions next to the antiphase contact with enhanced intensity due to the replacement of Mn by heavier La atoms. (d) Atomic model of the dislocation core. 
(a)

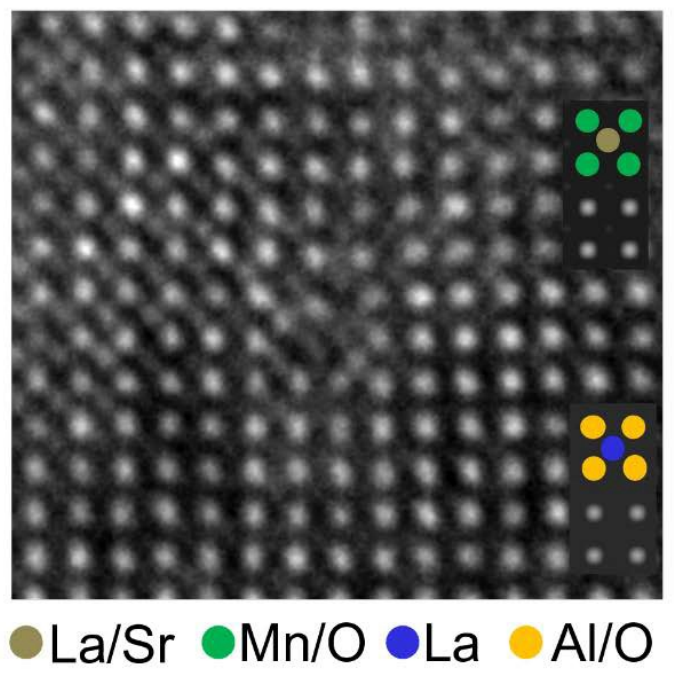

(b)

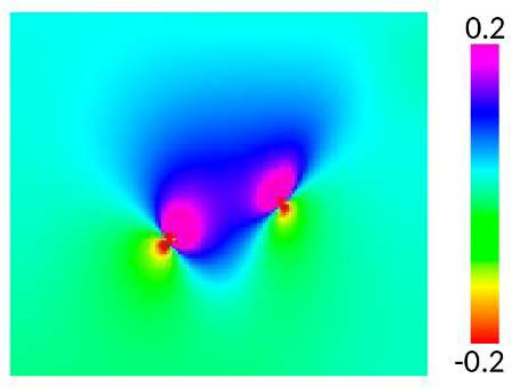

(c)

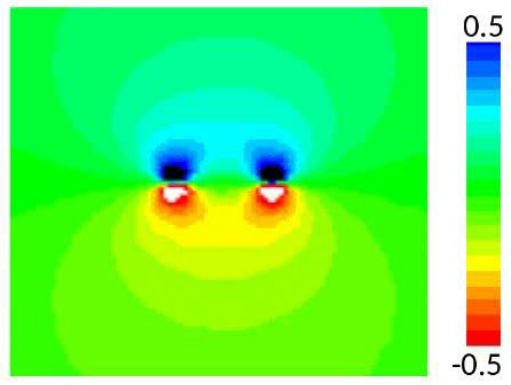

Figure 2. Structure and strain field of misfit dislocations in LSMO/LAO thin films. (a) HRTEM image of a misfit dislocation core viewed along the [100] zone axis. Insets are image simulations obtained for a defocus value of $75 \mathrm{~nm}$. The two arrows mark the two extra half planes of the dissociated core, nominally Al/O. (b) Dilatation map obtained from (a) by geometrical phase analysis. (c) Theoretical dilatation map of an identical configuration. 

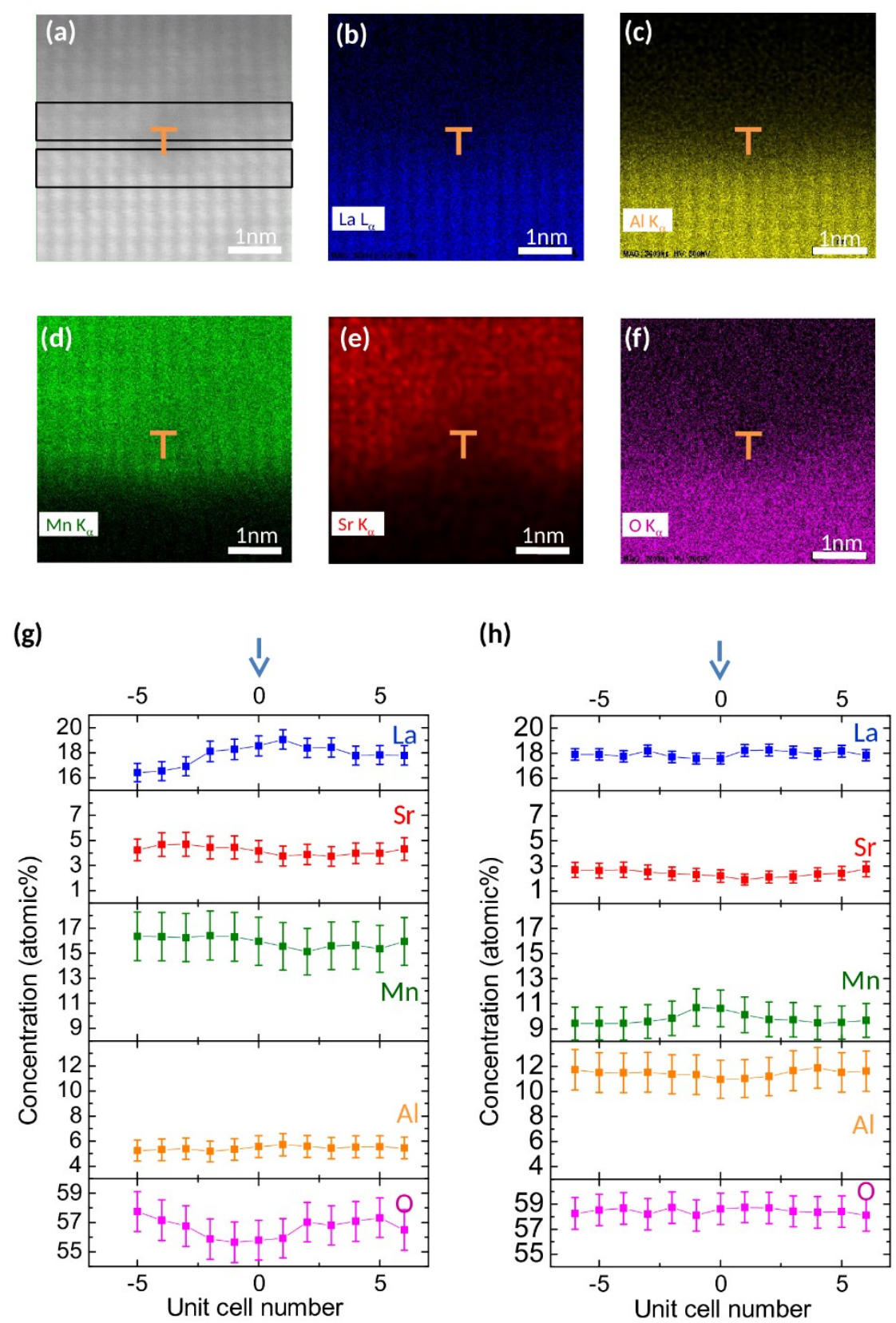

Figure 3. Elemental distribution around the misfit dislocation core. (a) Atomic resolution HAADF image of a misfit dislocation core, and associated EDX net count maps corresponding to (b) La-L, (c) Al-K, (d) Mn-K, (e) Sr-K, and (f) O-K ionization edges. (g) Elemental concentration horizontal profiles computed from (b)-(f) using the integration window above the glide plane as indicated in (a). (h) Elemental concentration horizontal 
profiles computed from (b)-(f) using the integration window below the glide plane as indicated in (a). Arrows mark the position of the axial plane of the misfit dislocation. 
(a)
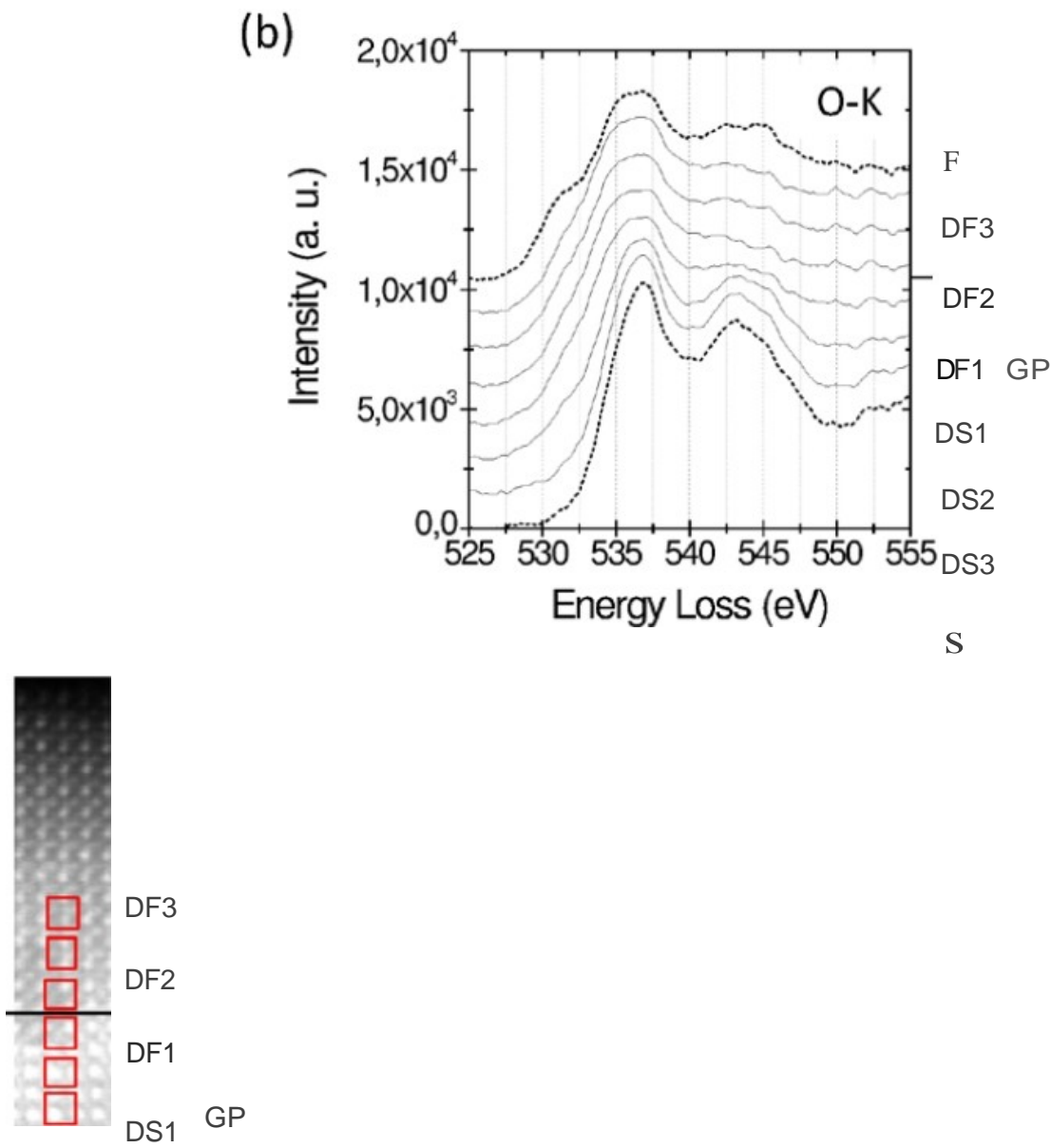

DS2 (c)

DS3

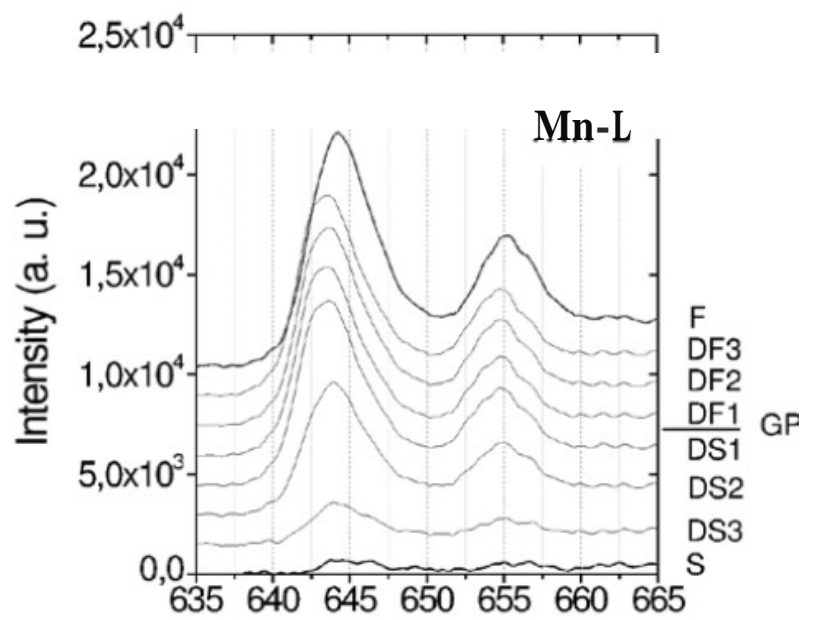


(d)

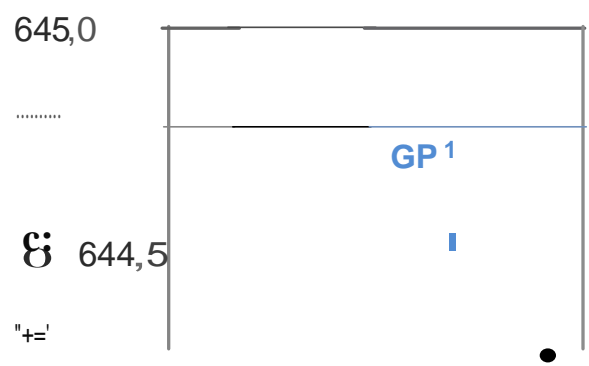

644,0

....: 643,5

Q)

0.

643,0 (e)

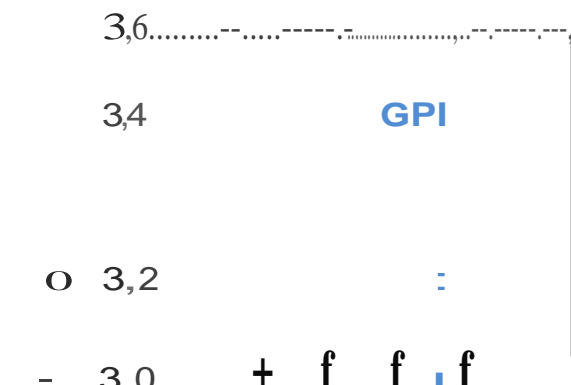

$0:: 2,8 \quad$ I $\quad$ f

Y 2,46-f--------

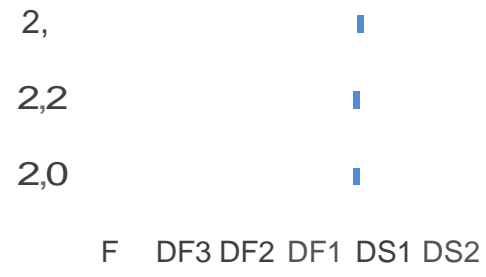


Figure 4. Vertical evolution of the EEL spectra across the misfit dislocation core. (a) Atomic resolution HAADF image, and simultaneously acquired $0-\mathrm{K}$ (b) and $\mathrm{Mn}-\mathrm{L}_{2,3}$ (c) EEL spectra from unit cells marked in (a) with red boxes. The glide plane (GP) is located between DFl and DSl as indicated in (a). (d), (e) Variation ofthe $\mathrm{L}_{3}$ peak position and the $\mathrm{L}_{2,3}$ intensity ratio, respectively. The glide plane is marked by a vertical dashed line. 
(a)

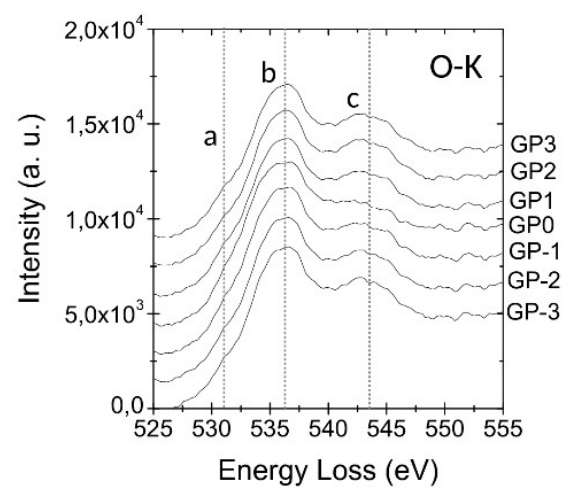

(b)

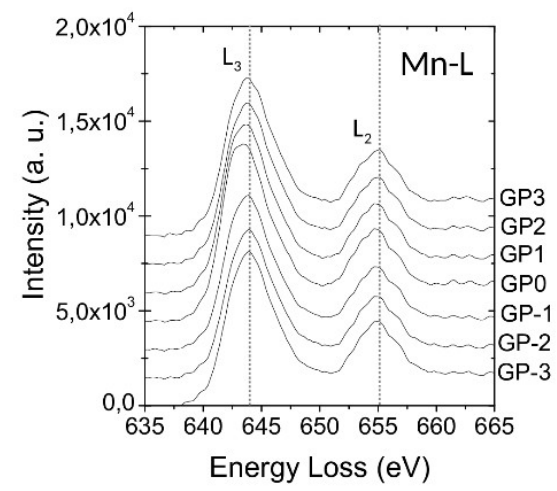

(c)

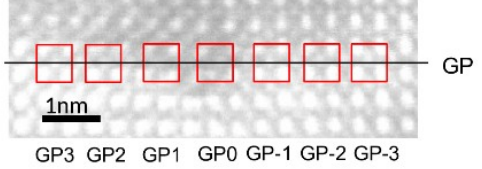

Figure 5. Lateral evolution of EEL spectra across the misfit dislocation core. (a) O-K edge and (b) $\mathrm{L}_{2,3}$ edge EEL spectra obtained from unit cells marked in the atomic resolution HAADF image shown in (c). GP0 corresponds to the locus of the misfit dislocation core. 


\section{(a)}

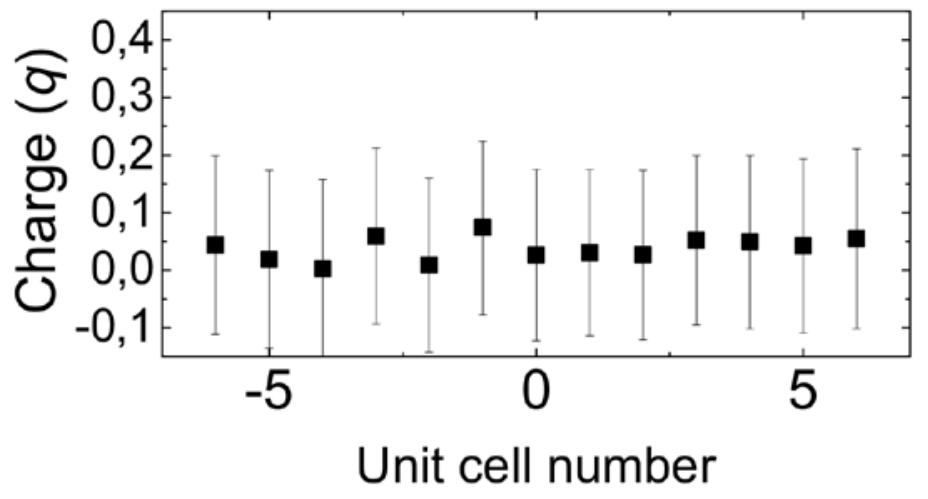

(b)

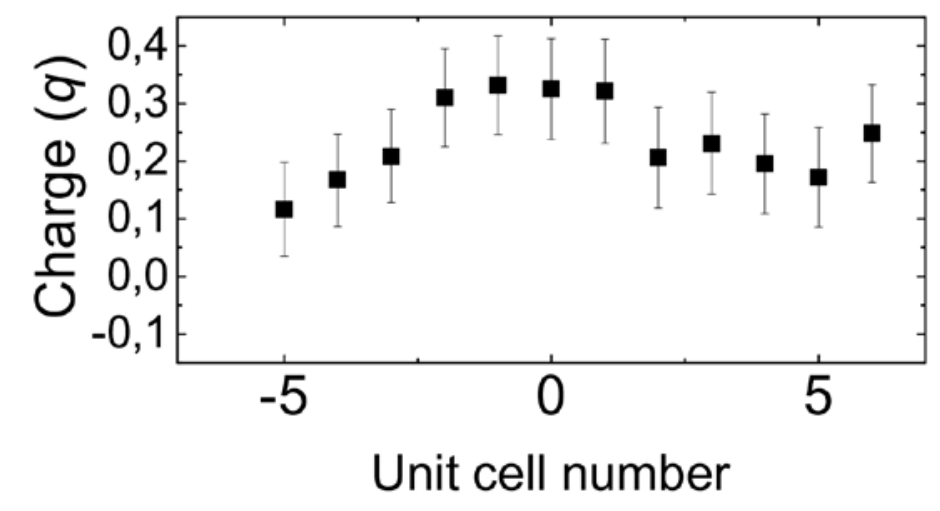

Figure 6. Unit cell charge characteristics computed from the ionic distributions below the glide (compressive zone) (a), and above the glide plane (tensile zone). 


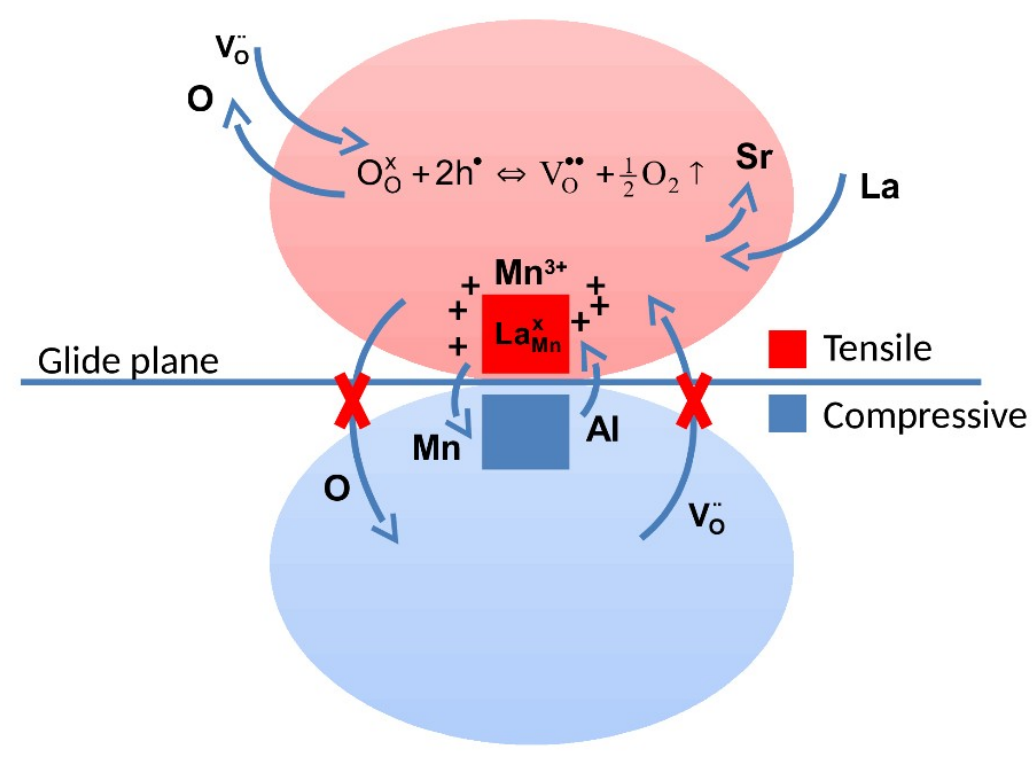

Figure 7. Schematic illustration of the basic mechanisms operating in the MD core. Red and blue represent tensile and compressive regions, respectively. The redox reaction indicated in the tensile region is displaced to the right, favoring the formation of electron donor oxygen vacancies, $\mathrm{V}_{0} \cdot$. Each vacancy nominally releases two electrons which can reduce two neighboring $\mathrm{Mn}^{4+}$ cations according to Equation (1). The inbalance between the rate of $\mathrm{Sr}$ diffusion out of the core region and the concentration of $\mathrm{V}_{0}^{*}$ results in a positive charge in the tensile region. The glide plane acts as a barrier for the redistribution of vacancies as indicated by crossed-out pathways. $\mathrm{La}_{\mathrm{Mn}}$ antisite defects form at the axial plane, on the tensile region, to accommodate the tensile strain. 
distortion showed a slight increase of the $L_{2,3}$. However, these variations in the simulated data 